Exotic Stars as Challenges to Evolution ASP Conference Series, Vol. 279, 2002

C. A. Tout and W. Van Hamme, eds.

\title{
Looking for Twins of Przybylski's Star
}

\author{
S. Hubrig
}

European Southern Observatory, Casilla 19001, Santiago 19, Chile

C. R. Cowley

Astronomy Department, University of Michigan, Ann Arbor, MI 48109-1090, USA

S. Bagnulo, G. Mathys

European Southern Observatory, Casilla 19001, Santiago 19, Chile

\section{A. Ritter}

Astrophysikalisches Institut Potsdam, An der Sternwarte 16, 14482 Potsdam, Germany

\section{G. M. Wahlgren}

Atomic Astrophysics, Lund Observatory, Box 43, 22100 Lund, Sweden

\begin{abstract}
The examination of high resolution spectra of two Ap stars, HD 965 and HD 217522, discloses a spectral appearance similar to Przybylski's star. Here we present the preliminary results of a comparative study of all three stars. Further, we discuss the possibility of having a good spectroscopic indicator of magnetic fields in Ap stars. In all three studied stars the strong LuII line at $\lambda 5984 \AA$ is present and very likely not affected by significant blends. Its hyperfine structure has been recently measured and we show the results of a model that accounts for the Paschen-Back effect on hyperfine structure.
\end{abstract}

\section{Introduction}

Przybylski's star (HD 101065, V816 Cen) is a pulsating chemically peculiar (roAp) star with a period of about $12.1 \mathrm{~min}$ and an amplitude $\Delta B_{\max }$ of 13 mmag (Kurtz \& Wegner 1979). The unusual nature of its spectrum was first noticed by Przybylski in 1961. This star has an extremely complex spectrum rich with lines of lanthanides which are so dominant that the presence of lines of irongroup elements has been controversial for almost twenty years. Usually, lines of neutral and singly-ionized iron are prominent in the spectra of Ap stars, and in some cases, the iron is clearly overabundant. In the star HD 101065, lines from neutral and singly-ionized elements of the iron group are weakly present, but so badly blended that Przybylski maintained they might not be present 
at all. Part of the controversy surrounding HD 101065 concerns its effective temperature. It has been shown that the structure of the atmosphere in this star is very unusual, giving rise to conflicting temperature estimates (Cowley et al. 2001). None of the standard-abundance Kurucz (1993) models can fit the observed colours and hydrogen line profiles, and effective temperatures in a very wide range are required to fit different features. Hence, definitive abundances cannot be determined until the puzzle about their atmospheric structure is clarified. The examination of high resolution spectra of two other Ap stars, HD 965 and HD 217522, discloses a spectral appearance similar to HD 101065. As for HD 101065, the strongest lines in the spectra of these stars belong to the lanthanides, although the higher line density in HD 101065 is immediately apparent. Both HD 101065 and HD 217522 are known rapidly oscillating Ap stars. No oscillations have been found for the star HD 965 so far. Nothing is known about the rotation periods of HD 101065 and HD 217522 - there is no rotational light variation, no measurable magnetic variation, no radial velocity variations or spectroscopic variability and no obvious rotational triplet in the pulsation amplitude spectrum. By contrast, the longitudinal field and the field modulus of HD 965 show a definite slow variation in observations spanning more than 5 years which is indicative of a rotation period greatly exceeding that value (Mathys et al. 2002). HD 101065 and HD 217522, which have not been observed so systematically, could also have very long periods. Alternatively, the absence of variations could also result from low inclination $i$ of the rotation axis to the line of sight, or of a small angle between the magnetic and rotation axes. Using considerations of Kurtz (1980) we can put a constraint on the orientation of HD 101065. Assuming a value $v \sin i=3.5 \mathrm{~km} \mathrm{~s}^{-1}$ (Cowley et al. 2000) and a radius of $R / R_{\odot}=1.8$ which corresponds to a $1.7 M_{\odot}$ star at the evolutionary stage indicated in the paper of Hubrig et al. (2000), we conclude that HD 101065 has a nearly pole-on orientation with $i=8: 6$. In this paper we wish to focus attention on the fundamental properties of all three peculiar stars.

\section{Comparative study}

\subsection{Identified species}

Observations were made on these three stars on two nights during 2000 May with the 8-m VLT and its UVES spectrograph at the resolution of 115000 and a signal-to-noise ratio larger than 400 . Detailed wavelength measurements and identifications are available only for HD 101065 (Cowley \& Mathys 1998). To show the similarities and differences in the spectral appearance between the three stars two spectral regions are presented in Fig. 1. It is easily seen that the richest spectra belong to the lanthanides in the first-ionization stage. However, third stages of $\mathrm{Pr}$ and Nd are also very strong in all three stars. The best identified spectra in HD 965 are those from Ce II, Nd II and Fe I. For the stars HD 101065 and HD 217522 the most prominent lines are from La II, Nd II, Sm II and Dy II. Quite a number of the strong unidentified lines that show up in the star HD 101065 are also present in HD 965 and HD 217522. Possibly some of these lines are Ce III lines. Unfortunately, for the third stages of the lanthanides which already become prominent in cool Ap stars, the necessary atomic data are only known for a small number of lines. A noticeable Zeeman broadening is 

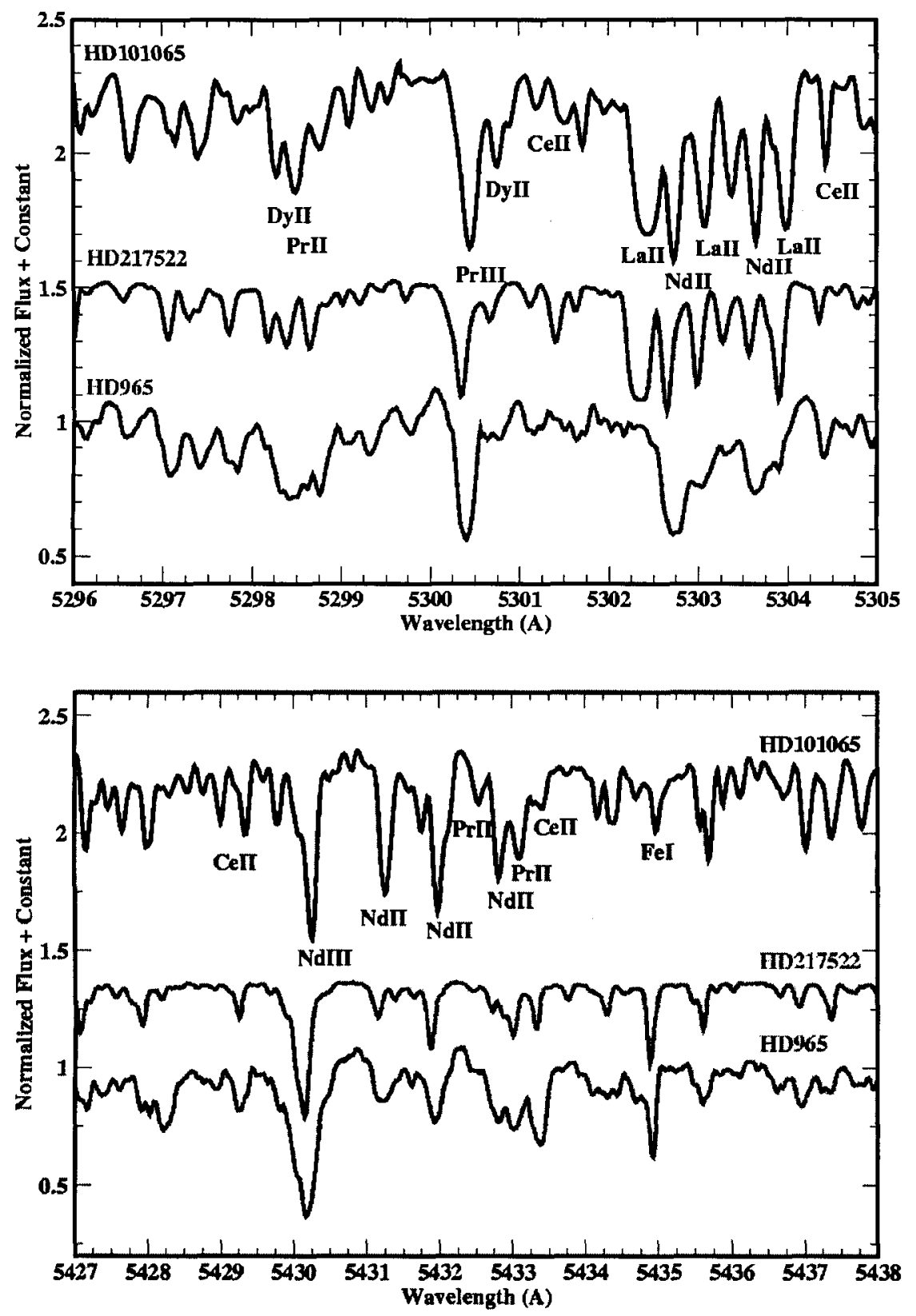

Figure 1. UVES observations of HD 101065, HD 217522 and HD 965 in two different spectral regions. The magnetically insensitive $\mathrm{Fe} I$ line at $\lambda 5434 \AA$ is sharp and has similar width in all three spectra. 


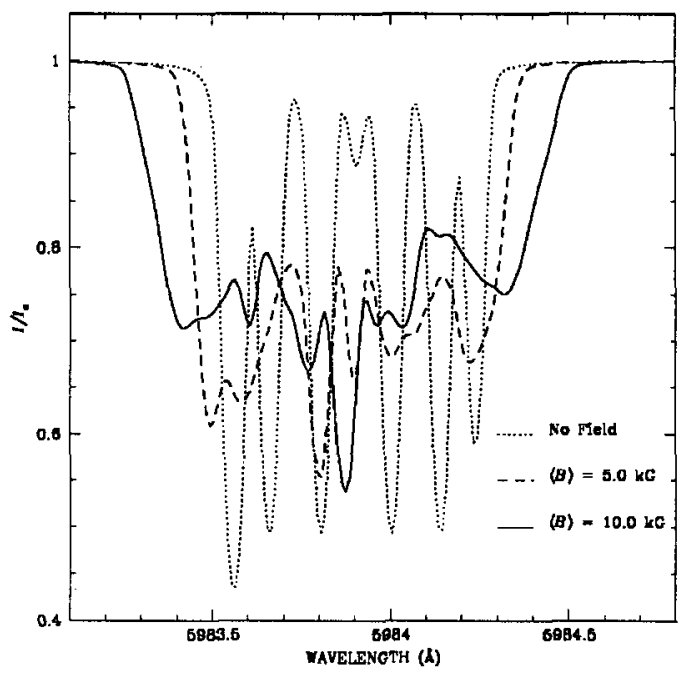

Figure 2. Synthetic spectra calculations for the line Lu II $\lambda 5984 \AA$ with the model atmosphere parameters $T_{\text {eff }}=7000 \mathrm{~K}, v \sin i=$ $0 \mathrm{~km} \mathrm{~s}^{-1}$ and for two different magnetic field strengths: $\langle H\rangle=5.0 \mathrm{kG}$ and $10 \mathrm{kG}$. The dotted line presents the line profile in absence of a magnetic field.

clearly visible in the spectrum of HD 965 . The magnetically insensitive $\mathrm{Fe} I$ line at $\lambda 5434 \AA$ is sharp and has a similar width in all three spectra. Therefore the value $v \sin i$ in all three stars should be around 3 or $3.5 \mathrm{~km} \mathrm{~s}^{-1}$. Virtually nothing is known about the spectral variability for all three stars. Our measurements of radial velocities in these three stars give values fully consistent with the previous determinations, i.e. $9.4 \mathrm{~km} \mathrm{~s}^{-1}$ for HD $101065,44.2 \mathrm{~km} \mathrm{~s}^{-1}$ for HD 217522 and $-0.7 \mathrm{~km} \mathrm{~s}^{-1}$ for HD 965 .

\subsection{Preliminary abundance analysis}

A number of cool magnetic chemically peculiar stars, among them HD 101065 , HD 965 and HD 217522, are known to exhibit abnormal profiles of hydrogen Balmer lines. This anomaly consists of a sharp transition between broad Stark wings and an unusually narrow Doppler core. This core-wing anomaly indicates a very abnormal structure of the atmosphere of cool magnetic CP stars. Recently, some preliminary results of an attempt to reproduce the core-wing anomaly by modification of the atmospheric temperature distribution have been reported (Cowley et al. 2001). In the paper by Cowley et al. (2000), it was argued that the observations of the star HD 101065 could be fitted, as a first approximation, by an extreme Ap star model with $T_{\text {eff }}=6600 \mathrm{~K}$ and $\log g=4.2$ with the allowance for enhanced blanketing. Using this model, elements with high abundances in the standard abundance distribution show depletions up 
to 1.5 dex and those with low abundances show enhancements about an order of some 3 dex. The temperature of all three stars is very hard to pin down, and conflicting results are obtained from the ionization, excitation, Balmer lines and Strömgren photometry. There is only one set of Strömgren photometry for HD 965 in SIMBAD and the values lead to the result $T_{\text {eff }}=6730 \mathrm{~K}$. Using Strömgren photometry for HD 217522 we obtain $T_{\text {eff }}=6808 \mathrm{~K}$. The $T_{\text {eff }}$ value derived from photometry for HD 965 is definitely too cool and calculations with a model using $T_{\text {eff }}=6730 \mathrm{~K}$ and $\log g=4$ produce $\mathrm{Fe} I$ and $\mathrm{Fe} I \mathrm{I}$ abundances which differ by over an order of magnitude. The most recent model has $T_{\text {eff }}=$ $8000 \mathrm{~K}, \log g=3$, and with no convection. With these parameters, the ionization discrepancy of $\mathrm{Fe} I$ and $\mathrm{Fe} I \mathrm{II}$ is $0.3 \mathrm{dex}$. At the moment it is not possible to describe these stars with a model that has uniform conditions at a fixed optical depth.

\subsection{Magnetic field}

Studies of magnetic fields of Ap stars are based on measurements of one or several of the following quantities: mean longitudinal magnetic field $\left\langle H_{z}\right\rangle$, crossover $v \sin i\left\langle x H_{z}\right\rangle$, mean quadratic magnetic field $\left(\left\langle H^{2}\right\rangle+\left\langle H_{z}^{2}\right\rangle\right)^{1 / 2}$, mean magnetic field modulus $\langle H\rangle$ and broad-band linear polarization. The most recent measurement of the longitudinal magnetic field in the star HD 101065 using a sample of $15 \mathrm{Nd}$ II lines, $\left\langle H_{z}\right\rangle=-1408 \pm 50 \mathrm{G}$, has been published by Cowley \& Mathys (1998). Cowley et al. (2000) report about a few lines of GdII and SmII in the spectrum of HD 101065 with large effective Landé factors showing partially resolved splitting in the spectrum. This splitting has been used to measure a magnetic field modulus of $2.3 \mathrm{kG}$. Using Fe I lines we have derived from the UVES spectrum a mean quadratic magnetic field $\left(\left\langle H^{2}\right\rangle+\left\langle H_{z}^{2}\right\rangle\right)^{1 / 2}=3106 \pm 1405 \mathrm{G}$. In the spectrum of HD 965 the Fe II line at $6149.2 \AA$ is resolved into magnetically split components while it is heavily blended with another line, probably with the Sm II line $(\lambda 6149.12 \AA)$, in the spectra of HD 101065 and HD 217522. The derived magnetic field modulus for HD $965\langle H\rangle=4.4 \mathrm{kG}$ is consistent with the measurements published by Mathys et al. (2002). Longitudinal magnetic field measurements for this star show a monotonic decrease over two years from $-574 \pm 113 \mathrm{G}$ to $-1270 \pm 139 \mathrm{G}$ (Mathys et al. 2002) The only determination of the magnetic field in HD 217522 has been published by Mathys \& Hubrig (1997) $\left\langle H_{z}\right\rangle=-394 \pm 124 \mathrm{G}$. One additional spectrum of this star has been taken with CASPEC at the 3.6-m telescope on La Silla in 1997. For the longitudinal field we have obtained $\left\langle H_{z}\right\rangle=-559 \pm 63 \mathrm{G}$. No crossover and quadratic magnetic field could be measured. From our recently obtained UVES spectrum we have derived the mean quadratic magnetic field $\left(\left\langle H^{2}\right\rangle+\left\langle H_{z}^{2}\right\rangle\right)^{1 / 2}=2017 \pm 403 \mathrm{G}$. In all three studied stars the strong line Lu II $\lambda 5984 \AA$ with a very broad pattern of hyperfine structure is present and probably can be used as a spectroscopic indicator of magnetic fields in Ap stars. This line is very likely not affected by significant blends. In the presence of magnetic fields, the effect of hyperfine structure can be remarkable in changing the Zeeman pattern, especially when the Zeeman splitting is smaller or comparable to the hyperfine structure splitting. In Fig. 2 we show the results of Lu II line profile modelling which account for the PaschenBack effect on the hyperfine structure. The line profiles have been computed with a radiative transfer code which makes use of the Milne-Eddington approx- 


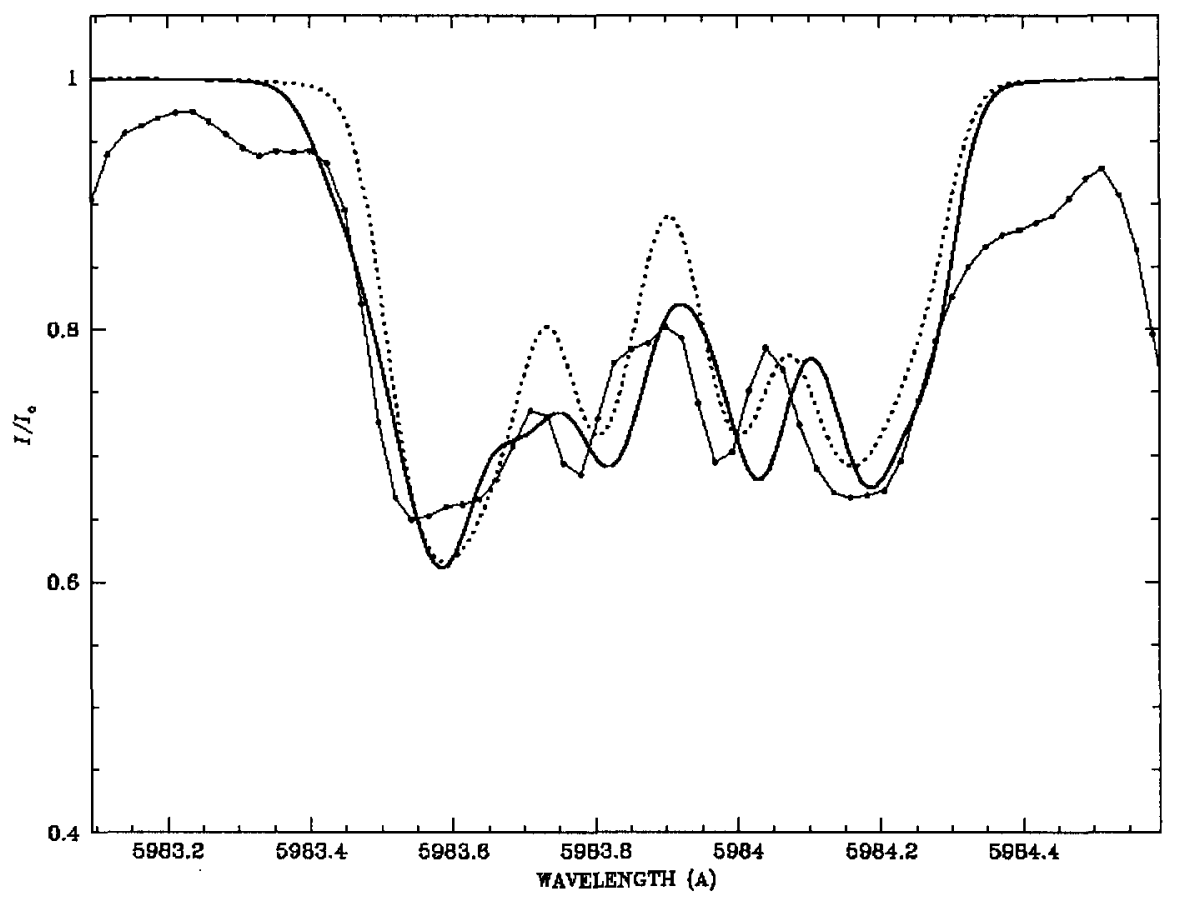

Figure 3. Comparison between the synthetic spectrum and observation for the line LuII $\lambda 5984 \AA$ in the spectrum of HD 101065. The synthetic spectrum is calculated with the model atmosphere parameters $T_{\text {eff }}=6600 \mathrm{~K}, v \sin i=3.5 \mathrm{~km} \mathrm{~s}^{-1}$ and $\langle H\rangle=3.0 \mathrm{kG}$ (solid line). The dotted line presents calculations without a magnetic field. Observations are indicated by dots connected with a solid line. 
imation. Our computations show that with an increasing strength of magnetic field the line profile of Lu II lines becomes shallower and broader. In Figs. 3 and 4 we present the calculations performed assuming appropriate atmosphere parameters for each of the studied stars. To improve the fit between the model profiles and the observed profiles we plan as a next step to incorporate in the calculations improved model parameters and to take possible blends into account.

\section{Discussion}

HD 101065 is most unusual among Ap stars with the most peculiar atmosphere known. Quite a number of the strong unidentified lines that show up in the spectrum of HD 101065 are also present in the spectra of HD 965 and HD 217522. However, we are not yet in a position to give definitive abundances. We need to find out if the line blanketing could possibly reconcile the colors with the apparent ionization temperature. We need more observations of the hydrogen lines to fix the gravity. We need higher resolution spectra, especially into the blue-violet, so we can see how deep the lines really get. Some strong lines of lanthanides, for example Ho II lines, are short of the region where our identification list starts, so we actually cannot say from our material that some elements are strong in the spectra of our stars. Of particular interest will be additional magnetic field measurements, as well as to have more photometry, to know if there are rapid oscillations in HD 965.

\section{References}

Cowley, C. R., Hubrig, S., Ryabchikova, T. A., Mathys, G., Piskunov, N., \& Mittermayer, P. 2001, A\&A, 367, 939

Cowley, C. R., \& Mathys, G. 1998, A\&A, 339, 165

Cowley, C. R., Ryabchikova, T., Kupka, F., Bord, D. J., Mathys, G., \& Bidelman, W. P. 2000, MNRAS, 317, 299

Hubrig, S., Kharchenko, N., Mathys, G., \& North, P. 2000, A\&A, 355, 306

Kurucz, R. L. 1993, Kurucz CD-ROM No. 2 \& No. 13 (Cambridge: SAO)

Kurtz, D. W. 1980, MNRAS, 191, 115

Kurtz, D. W., \& Wegner, G. 1979, ApJ, 232, 510

Mathys, G., \& Hubrig, S. 1997, A\&AS, 124, 475

Mathys, G., Manfroid, J., \& Wenderoth, E. 2002, in preparation 

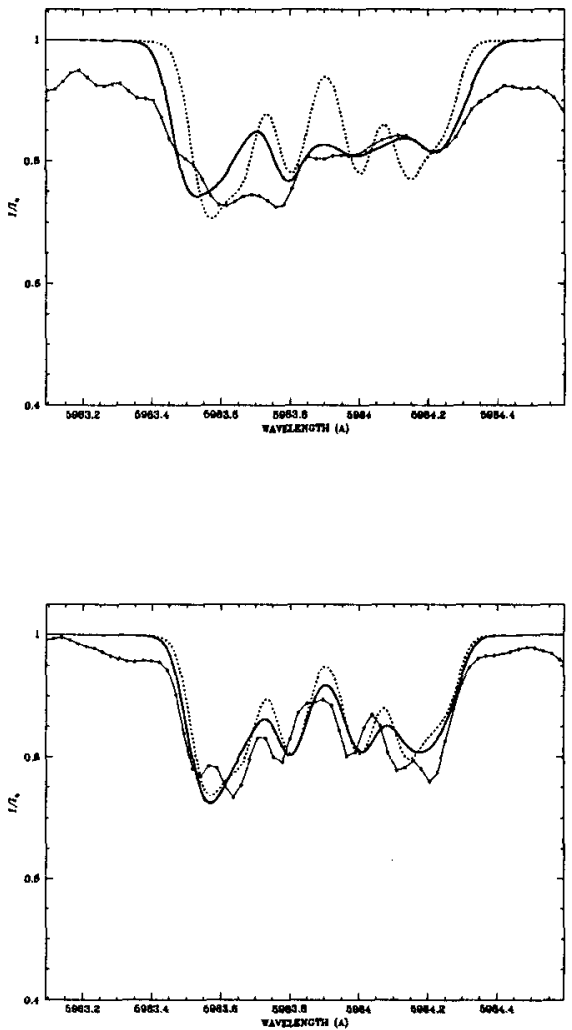

Figure 4. Comparison between the synthetic spectrum and observation for the line LuII $\lambda 5984 \AA$ in the spectra of HD 965 and HD 217522. The synthetic spectrum for HD 965 (left) is calculated with the model atmosphere parameters $T_{\text {eff }}=8000 \mathrm{~K}, v \sin i=3.0 \mathrm{~km} \mathrm{~s}^{-1}$ and $\langle H\rangle=4.5 \mathrm{kG}$ (solid line). For HD 217522 (right) the model atmosphere parameters are $T_{\text {eff }}=6750 \mathrm{~K}, v \sin i=3.0 \mathrm{~km} \mathrm{~s}^{-1}$ and $\langle H\rangle=2.0 \mathrm{kG}$ (solid line). The dotted line presents calculations without magnetic field. Observations are indicated by dots connected with a solid line. 